OPEN ACCESS

Edited by:

Shijun $\mathrm{Hu}$,

Soochow University, China

Reviewed by:

Yongming Wang,

Fudan University, China

Lin Liu,

Nankai University, China

*Correspondence:

Won Hee Lee

whlee@email.arizona.edu

Yoon-Goo Kim

ygkim26@skku.edu

tThese authors have contributed equally to this work and share first

authorship

Specialty section:

This article was submitted to

Stem Cell Research,

a section of the journal

Frontiers in Cell and Developmental

Biology

Received: 18 October 2020

Accepted: 08 December 2020

Published: 12 January 2021

Citation:

Jang HR, Cho HJ, Zhou Y, Shao N-Y,

Lee K, Le HHT, Jeon J, Lee JE,

Huh W, Ong S-G, Lee WH and

Kim Y-G (2021) Modeling Uremic

Vasculopathy With Induced

Pluripotent Stem Cell-Derived

Endothelial Cells as a Drug Screening

System.

Front. Cell Dev. Biol. 8:618796.

doi: 10.3389/fcell.2020.618796

\title{
Modeling Uremic Vasculopathy With Induced Pluripotent Stem Cell-Derived Endothelial Cells as a Drug Screening System
}

\begin{abstract}
Hye Ryoun Jang ${ }^{1 \dagger}$, Hyung Joon $\mathrm{Cho}^{2+}$, Yang Zhou ${ }^{3}$, Ning-Yi Shao ${ }^{4}$, Kyungho Lee ${ }^{1}$, Hoai Huong Thi Le ${ }^{5}$, Junseok Jeon ${ }^{1}$, Jung Eun Lee ${ }^{1}$, Wooseong Huh ${ }^{1}$, Sang-Ging Ong ${ }^{6,7}$, Won Hee Lee ${ }^{5 *}$ and Yoon-Goo Kim ${ }^{1 *}$
\end{abstract}

\begin{abstract}
${ }^{1}$ Division of Nephrology, Department of Medicine, Samsung Medical Center, Stem Cell \& Regenerative Medicine Institute(SCRMI), Sungkyunkwan University School of Medicine, Seoul, South Korea, ${ }^{2}$ School for Engineering of Matter, Transport \& Energy, Arizona State University, Tempe, AZ, United States, ${ }^{3}$ Stanford Cardiovascular Institute, Stanford University School of Medicine, Stanford, CA, United States, ${ }^{4}$ Health Sciences, University of Macau, Macau, China, ${ }^{5}$ Department of Basic Medical Sciences, University of Arizona College of Medicine, Phoenix, AZ, United States, ${ }^{6}$ Department of Pharmacology \& Regenerative Medicine, University of Illinois College of Medicine, Chicago, IL, United States, ${ }^{7}$ Division of Cardiology, Department of Medicine, University of Illinois College of Medicine, Chicago, IL, United States
\end{abstract}

Background: Cardiovascular complications are the leading cause of mortality in patients with chronic kidney disease (CKD). Uremic vasculopathy plays a crucial role in facilitating the progression of cardiovascular complications in advanced CKD. However, the improvement of conventional research methods could provide further insights into CKD.

Objectives: In this study, we aimed to develop a novel model of uremic vasculopathy as a potential drug screening system.

Methods and Results: The effects of uremic serum and different combinations of uremic toxins on induced pluripotent stem cell (iPSC)-derived endothelial cells (ECs) of a normal control and a CKD patient were investigated using several functional assays. We found that a mixture of uremic toxins composed of high urea, creatinine, uric acid, and indoxyl sulfate exerted deleterious effects on normal control iPSC-ECs that were comparable to uremic serum by increasing reactive oxygen species and apoptosis, as well as suppression of tube formation. Additional characterization revealed a potential involvement of dysregulated TGF- $\beta$ signaling as treatment with either losartan or TGF- $\beta$ inhibitors led to the attenuation of adverse effects induced by uremic toxins. Importantly, impaired wound healing potential seen in CKD patient-specific iPSC-ECs was rescued by treatment with losartan and TGF- $\beta$ inhibitors.

Conclusion: Our study demonstrated that simplified uremic toxin mixtures can simulate the uremic micromilieu reproducibly and CKD patient-specific iPSC-ECs can potentially recapitulate susceptibility to uremic vasculopathy. This novel model of uremic vasculopathy may provide a new research tool as a drug screening system.

Keywords: endothelial cells, induced pluripotent stem cells, uremic vasculopathy, uremic toxin, chronic kidney disease 


\section{INTRODUCTION}

Cardiovascular complications are the leading cause of mortality and major morbidities in patients with chronic kidney disease (CKD) (Go et al., 2004; Tonelli et al., 2006; Stenvinkel, 2010). Uremic vasculopathy is the cornerstone of cardiovascular complications acting as the primary factor that accelerates atherosclerosis and heart failure (Levin et al., 1990; Deppisch et al., 2001; Jourde-Chiche et al., 2011; Sallée et al., 2014). It also remains an important hurdle for kidney transplantation in endstage renal disease (ESRD) patients undergoing longstanding dialysis by causing severe atherosclerosis and heavy vascular calcification (Jourde-Chiche et al., 2011; Torres and de Broe, 2012). Although epidemiologic research can provide insightful results from CKD patients in the real world, large sample sizes with very long follow-up periods are required and the results can often be confounded by factors such as comorbidities other than CKD (Ballew and Matsushita, 2018). Therefore, studies revealing the pathophysiology of uremic vasculopathy and investigation of potential new drugs or the adequacy of current drugs are required to improve overall outcomes for CKD patients.

Previous studies using genetically engineered or surgically simulated murine CKD models have provided significant insights into the pathophysiology of uremia-induced cardiovascular complications (Baraka and El Ghotny, 2012; Xie et al., 2015). However, these murine models have limitations for investigating the effects of uremia on vessels because of interspecies differences and methodological limitations in simulating CKD or evaluating changes in vessels. To investigate the pathophysiology of uremic vasculopathy thoroughly in the hope of identifying new drugs, a novel model of uremic vasculopathy with human endothelial cells (ECs) that reflects genetic susceptibility to uremic toxins is essential.

In this study, we developed a novel model of uremic vasculopathy using ECs differentiated from human induced pluripotent stem cells (iPSCs). The effects of several uremic toxins on iPSC-ECs were compared with those of uremic serum from ESRD patients. The feasibility of this new model as a novel drug screening tool was also investigated.

\section{MATERIALS AND METHODS \\ Study Participants and Design}

Serum and peripheral blood mononuclear cells (PBMCs) were collected from 5 healthy volunteers and 5 ESRD patients receiving hemodialysis for more than a year. Informed consent was obtained from all subjects. The Institutional Review Board of the Samsung Medical Center approved the study protocol in compliance with the Declaration of Helsinki (October 2008), and informed consent was waived because of the retrospective and non-interventional design of the study (IRB number: 2016-11-025).

Several functional assays for comparing the effects of serum from normal controls and ESRD patients as well as simplified uremic toxin mixtures on iPSC-ECs were performed for establishing a new model of uremic vasculopathy.
The wound healing potential of normal control iPSC-ECs and ESRD patient-specific iPSC-ECs was also compared by a scratch migration assay.

\section{Generation of iPSC-ECs}

PBMCs from a normal control and an ESRD patient were reprogrammed into iPSCs using Sendai virus (Takahashi et al., 2007; Churko et al., 2013). ECs were differentiated from iPSCs according to the protocol validated in previous studies (Sa et al., 2017; Bezenah et al., 2018; Lee et al., 2019). Briefly, the iPSCs were treated with a differentiation induction medium (RPMI and B-27 minus insulin, Thermo Fisher Sci, Waltham, MA) supplemented with $6 \mu \mathrm{M}$ and $2 \mu \mathrm{M}$ of glycogen synthase kinase 3- $\beta$ inhibitor, CHIR-99021 (Selleck Chemicals, Houston, TX), on day 0 and day 2 , respectively. From day 4 to 12 of differentiation, cells were cultured in different combinations of differentiation induction media and EGM-2 media from Lonza (100\% differentiation medium on day $4,50 \%$ differentiation and 50\% EGM-2 media on day $6,25 \%$ differentiation and $75 \%$ EGM2 media on day 8, and $100 \%$ EGM2 medium on day 10) with growth factors including $50 \mathrm{ng} / \mathrm{ml} \mathrm{VEGF,} 20 \mathrm{ng} / \mathrm{ml} \mathrm{FGF} 2$, and $20 \mathrm{ng} / \mathrm{ml} \mathrm{BMP} 4$ (PeproTech, Rocky Hill, NJ). On day 12 post-differentiation, cells were sorted by a magnetic cell sorting (MACS) system (Miltenyi Biotech, San Diego, CA) using magnetic beads conjugated to human CD144 antibodies, as directed by the manufacturer, and expanded on $0.2 \%$ gelatin-coated plates. iPSC-ECs were then cultured in EGM2 medium at $37^{\circ} \mathrm{C}$ and $5 \% \mathrm{CO}_{2}$ in a humidified incubator with medium changes every other day. The experiments described in this manuscript were performed between passages 2-4. The phenotypes of iPSC-ECs used in this study were characterized in previous studies (Lee et al., 2019; Ong et al., 2019).

\section{Serum Sample Preparation and Analytic Procedure}

For serum preparation, blood was separated by centrifuging clotted blood at 3,500 rpm for $15 \mathrm{~min}$ at $4^{\circ} \mathrm{C}$ and was aliquoted before storing at $-80^{\circ} \mathrm{C}$ until needed for experiments. Serum uric acid, creatinine, and blood urea nitrogen were measured using Fuji Dri-Chem 7000i (Fujifilm Corporation, Tokyo, Japan).

\section{Preparation of Uremic Toxin Mixtures}

Urea, creatinine, indoxyl sulfate (Sigma-Aldrich, Louis, MO), and advanced glycation end-products (AGEs, BioVision, San Francisco, CA) were dissolved in Dulbecco's phosphate-buffered saline and uric acid (Sigma-Aldrich) was dissolved in $1 \mathrm{M}$ $\mathrm{NaOH}$, then the solutions were filtered $(0.22 \mu \mathrm{m}$ pore size $)$ before experiments.

\section{Luminex Multiplex Assay}

Serum samples were mixed with antibody-linked polystyrene beads on 96-well filter plates and incubated at room temperature for $2 \mathrm{~h}$, followed by overnight incubation at $4{ }^{\circ} \mathrm{C}$ on an orbital shaker at 500-600 rpm. After washing plates twice with wash buffer, a biotinylated detection antibody was added for $2 \mathrm{~h}$ at room temperature with shaking. Samples were then filtered, washed twice, and resuspended in streptavidin-PE for $40 \mathrm{~min}$ 
at room temperature. Two additional vacuum washes were performed before adding the reading buffer. All standards and samples were measured in duplicate. Plates were read using a Luminex 200 instrument with a lower bound of 100 beads per sample per cytokine. For quality control, custom assay control beads (Radix Biosolutions, Georgetown, TX) were added to all wells.

\section{Assays for Cellular Viability, Reactive Oxygen Species (ROS), and Caspase 3/7 Activity}

iPSC-ECs plated on 96-well plates were subjected to plate-based assays after serum (15\%) or uremic toxin mixture treatments. Assays using CellTiter-Glo 2.0, ROS-Glo $\mathrm{H}_{2} \mathrm{O}_{2}$, and CaspaseGlo 3/7 (Promega, Madison, WI) were performed following the manufacturer's instruction and the luminescence signal was recorded on the GloMax-Multi detection system (Promega). Cells in the original sample plate were kept for measuring the total cell number by calcein-AM (Thermo Fisher Scientific) to allow normalization.

\section{Measurement of Endothelial Function}

An in vitro endothelial tube formation assay was carried out following the manufacturer's instructions. Briefly, after coating the 15-well $\mu$-Slice Angiogenesis (Ibidi GmbH, Gräfelfing, Germany) with Corning Matrigel Basement Membrane Matrix, iPSC-ECs pre-treated with serum or uremic toxin mixture (UT)$\mathrm{H}$ with or without drugs for $48 \mathrm{~h}$ were seeded at $1 \times 10^{4}$ cells/well. After $16 \mathrm{~h}$ incubation, capillary network images were taken using a Revolve microscope and quantitation was made using ImageJ.

For the in vitro migration assay, normal control iPSC-ECs and ESRD patient-specific iPSC-ECs were treated with drugs for $48 \mathrm{~h}$ before seeding in 24 -well plate $\left(5 \times 10^{4}\right.$ cells/well). When cells reached $80 \%$ confluence, the culture medium was replaced overnight for serum starvation. A straight line was scraped in the cell monolayer with a sterile $200 \mu \mathrm{l}$ pipette tips and the debris was removed by washing twice with media. Cells were then incubated with EGM2 media and imaged at 0 , 4, and $10 \mathrm{~h}$ after the scratch. The area and width of the scratch were analyzed with ImageJ. Losartan (a well-known angiotensin-receptor blocker; Sigma), SD-208 (a selective TGF- $\beta$ receptor I kinase (ALK5) inhibitor; Selleckchem, Houston, TX), and LY2109761 (TGF- $\beta$ receptor I and type II dual inhibitor; Selleckchem) were used for drug treatment.

\section{Human TGF- $\beta$ Pathway Protein Phosphorylation}

After treating iPSC-ECs with either the control or UT-H for $24 \mathrm{~h}$, the relative levels of phosphorylation of 8 TGF- $\beta$ pathway proteins in cell lysates were quantified using human TGF- $\beta$ pathway phosphorylation array (C1 series, RayBiotech, Peachtree Corners, GA) following the manufacturer's instructions. Briefly, after blocking each well, samples were incubated for $2 \mathrm{~h}$ at room temperature with membranes arrayed with antibodies against 8 TGF- $\beta$ phosphorylated proteins. After washing twice in wash buffer, a detection antibody cocktail was added into each well for $2 \mathrm{~h}$ at room temperature. After washing, membranes were then incubated with horseradish peroxidase (HRP)-rabbit IgG for $2 \mathrm{~h}$, washed, and placed in the chemiluminescence detection buffer. FluorChem E (Protein Simple, San Jose, CA) was used to detect signal chemiluminescence intensities from protein array membranes. Quantitative analysis for chemiluminescent intensity was conducted using Image J.

\section{RNA-seq Library Preparation and Analysis}

Total RNA was first extracted from iPSC-ECs using a RNeasy Mini Kit (Qiagen, Hilden, Germany) according to the manufacturer's instruction and $100 \mathrm{ng}$ RNA was used to construct sequencing libraries. Libraries were prepared using the TruSeq Stranded Total RNA Library Prep Kit (Illumina, San Diego, CA) and the raw 150 bp paired-end RNA-seq reads sequenced by Illumina HiSeq 2000 were trimmed by TrimGalore version 0.4.2 to exclude adapter sequences and bases with Phred scores of $<20$, providing a low probability of base-calling error $(p<$ $1 \%$ ) equivalent to $>99 \%$ accuracy. The reads were aligned to the human genome hg38 by Hisat2 (Pertea et al., 2016), and annotated by FeatureCounts of the Subread package (Liao et al., 2014). Differentially expressed genes were detected by DESeq2 (Love et al., 2014). The gene ontology enrichment analyses were implemented by Metascape (Zhou et al., 2019).

\section{Statistical Analyses}

Data are expressed as the mean \pm standard error of the mean (SEM) or mean \pm standard deviation (SD). Group means were compared with the Mann-Whitney test using SPSS $12.0 \mathrm{~K}$ or analysis of variance (ANOVA) followed by Newman-Keuls post hoc analysis using GraphPad Prism version 8 (GraphPad, San Diego, CA). Statistical significance was determined when the $P$-value was $<0.05$.

\section{RESULTS}

\section{Uremic Toxin Mixtures Mimic the Effects of Uremic Serum on iPSC-ECs}

To establish a baseline for studying uremic vasculopathy, we first examined the effects of uremic serum collected from ESRD patients or normal serum from healthy volunteers on iPSCECs. Several parameters including cell viability, generation of reactive oxygen species (ROS), and apoptosis were measured following exposure of iPSC-ECs to uremic or normal serum. The blood urea nitrogen and serum creatinine concentrations of uremic serum collected immediately before hemodialysis are summarized with the baseline characteristics of ESRD patients in Table 1. The mean duration of dialysis was 9.8 years and hypertension was present in all ESRD patients.

iPSC-ECs treated with uremic serum exhibited a significant decrease in cell viability (Figure 1A) accompanied by a significant increase in ROS generation measured by $\mathrm{H}_{2} \mathrm{O}_{2}$ production (Figure 1B) compared to iPSC-ECs exposed to normal serum. In addition, the degree of apoptosis measured by caspase 3/7 activity in iPSC-ECs treated with uremic serum was not discernible at $4 \mathrm{~h}$ compared to those cultured in normal serum but was significantly enhanced by $24 \mathrm{~h}$ post-treatment 
TABLE 1 | Baseline characteristics of normal controls and ESRD patients.

\begin{tabular}{|c|c|c|c|}
\hline Characteristics & $\begin{array}{c}\text { Normal } \\
\text { controls }(n=5)\end{array}$ & $\begin{array}{l}\text { ESRD patients } \\
\quad(n=5)\end{array}$ & $P$-value \\
\hline Age, years & $32 \pm 7.6$ & $49 \pm 11.1$ & 0.0317 \\
\hline Male: female & 3: 2 & 3: 2 & $>0.9999$ \\
\hline $\mathrm{BMI}\left(\mathrm{kg} / \mathrm{m}^{2}\right)$ & $23.9 \pm 4.43$ & $24.5 \pm 6.52$ & $>0.9999$ \\
\hline $\begin{array}{l}\text { Blood urea nitrogen } \\
(\mathrm{mg} / \mathrm{dL})\end{array}$ & $12.48 \pm 2.119$ & $51.04 \pm 11.32$ & 0.0079 \\
\hline $\begin{array}{l}\text { Serum creatinine } \\
(\mathrm{mg} / \mathrm{dL})\end{array}$ & $0.62 \pm 0.110$ & $10.44 \pm 3.480$ & 0.0079 \\
\hline $\begin{array}{l}\text { Serum uric acid } \\
(\mathrm{mg} / \mathrm{dL})\end{array}$ & $5.24 \pm 1.322$ & $7.72 \pm 2.100$ & 0.0635 \\
\hline Causes of ESRD, $n(\%)$ & NA & & NA \\
\hline Diabetes mellitus & & $1(20 \%)$ & \\
\hline Hypertension & & $1(20 \%)$ & \\
\hline Glomerulonephritis & & $2(40 \%)$ & \\
\hline ADPKD & & $1(20 \%)$ & \\
\hline $\begin{array}{l}\text { Cardiovascular } \\
\text { diseases, } n(\%)\end{array}$ & NA & & NA \\
\hline Hypertension & & 5 (100\%) & \\
\hline Pericardial effusion & & $2(40 \%)$ & \\
\hline Diastolic dysfunction & & $1(20 \%)$ & \\
\hline Aortic dissection & & $1(20 \%)$ & \\
\hline $\begin{array}{l}\text { Duration of } \\
\text { hemodialysis, years }\end{array}$ & NA & $9.8(2-15)$ & NA \\
\hline
\end{tabular}

Laboratory values are mean \pm standard deviation.

$A D P K D$, autosomal dominant polycystic kidney disease; BMI, body mass index; ESRD, end-stage renal disease; NA, not applicable.

(Figure 1C). Collectively, these results demonstrated that iPSCECs exposed to uremic serum collected from ESRD patients exhibited hallmarks of endothelial dysfunction.

Having demonstrated that uremic serum induces endothelial dysfunction in iPSC-ECs, we then sought to identify a minimal in vitro mixture of uremic toxins that would elicit similar effects on iPSC-ECs. To achieve this, we tested 25 combinations of substances known to be present in uremic serum (Table 2) based on three parameters: cell viability, ROS production, and apoptotic activity. We first compared control conditions and found that there were no significant differences in cell viability, ROS levels, and caspase $3 / 7$ activity between control (CTL) 1 consisting of media only and CTL4 (media containing physiological levels of urea, creatinine, and uric acid). Subsequent testing revealed that compared with CTL1 and CTL4, exposure of iPSC-ECs in two different combinations of uremic toxin mixture, namely UT6 (containing a high concentration of urea, creatinine, uric acid, indoxyl sulfate, and AGE) and UT-H (containing a high concentration of urea, creatinine, uric acid, and indoxyl sulfate) led to significantly lower cell viability (Figure 1D). Likewise, both ROS production (Figure 1E) and apoptosis (Figure 1F) were significantly elevated in cells exposed to UT6 and UT-H.

\section{Effects of Uremic Serum and Uremic Toxin Mixtures on the Angiogenetic Ability of iPSC-ECs}

We next sought to explore how uremic serum or uremic toxin mixture affect the angiogenic capability of iPSC-ECs. As shown
TABLE 2 | Composition of in vitro uremic toxin mixtures.

\begin{tabular}{|c|c|c|c|c|c|c|}
\hline Groups & $\begin{array}{l}\text { Urea, mM } \\
\text { (BUN, } \\
\text { mg/dL) }\end{array}$ & $\begin{array}{c}\text { Creatinine, } \\
\text { mM } \\
(\mathrm{mg} / \mathrm{dL})\end{array}$ & $\begin{array}{c}\text { Uric acid, } \\
\mathrm{mM} \\
(\mathrm{mg} / \mathrm{dL})\end{array}$ & $\begin{array}{c}\text { Indoxyl } \\
\text { sulfate } \\
\text { (mM) }\end{array}$ & $\begin{array}{c}\text { AGE } \\
(\mathrm{mg} / \mathrm{L})\end{array}$ & $\underset{(\mu \mathrm{L} / \mathrm{mL})}{\mathrm{NaOH}}$ \\
\hline \multicolumn{7}{|c|}{ Control 1 (media only) } \\
\hline Control 2 & $5(14.01)$ & $0.1(1.13)$ & & & & \\
\hline Control 3 & $5(14.01)$ & $0.1(1.13)$ & $0.25(4.20)$ & & & 11 \\
\hline Control 4 & $5(14.01)$ & $0.1(1.13)$ & $0.25(4.20)$ & & & \\
\hline Control 5 & & & & & & 16 \\
\hline UA 1 & & & $0.5(8.41)$ & & & \\
\hline UA 2 & & & $0.8(13.45)$ & & & \\
\hline UA 3 & & & $1(16.81)$ & & & \\
\hline Urea & $50(142.86)$ & & & & & \\
\hline Creatinine & & $1(11.30)$ & & & & \\
\hline IS & & & $1(16.81)$ & & & \\
\hline UT 1 & $50(142.86)$ & $1(11.30)$ & & & & \\
\hline UT 2 & $50(142.86)$ & $1(11.30)$ & $1(16.81)$ & & & \\
\hline UT 3 & $50(142.86)$ & $1(11.30)$ & $1(16.81)$ & 0.5 & & \\
\hline UT 4 & $50(142.86)$ & $1(11.30)$ & $1(16.81)$ & 0.5 & 1 & \\
\hline UT 5 & $25(71.43)$ & $1(11.30)$ & $1(16.81)$ & 0.5 & 10 & \\
\hline UT 6 & $50(142.86)$ & $1(11.30)$ & $1(16.81)$ & 0.5 & 10 & \\
\hline UT-A & $25(71.43)$ & $1(11.30)$ & $0.5(8.41)$ & & & \\
\hline UT-B & $25(71.43)$ & $1(11.30)$ & $1(16.81)$ & & & \\
\hline UT-C & $50(142.86)$ & $1(11.30)$ & $0.5(8.41)$ & & & \\
\hline UT-D & $50(142.86)$ & $1(11.30)$ & $1(16.81)$ & & & \\
\hline UT-E & $25(71.43)$ & $1(11.30)$ & $0.8(13.45)$ & & & \\
\hline UT-F & $25(71.43)$ & $1(11.30)$ & $0.8(13.45)$ & 1 & & \\
\hline UT-G & $50(142.86)$ & $1(11.30)$ & $0.8(13.45)$ & & & \\
\hline UT-H & $50(142.86)$ & $1(11.30)$ & $0.8(13.45)$ & 1 & & \\
\hline
\end{tabular}

AGE, advanced glycation end-products.

in Figures 2A,B we observed that uremic serum significantly suppressed tube formation of iPSC-ECs determined by the number of nodes and meshes and the total area of meshes compared to normal serum. Similar findings were also revealed in iPSC-ECs supplemented with UT-H mimicking the effect of uremic serum. High concentrations of urea, uric acid, or indoxyl sulfate consistently suppressed tube formation of iPSCECs (Figure 3).

\section{Activation of Intracellular TGF- $\beta$ Pathway in IPSC-ECs by in vitro Uremic Toxin Mixtures and the Different Expression of Inflammatory Cytokines in Uremic Serum}

TGF- $\beta$ has been implicated as a major regulatory cytokine playing important and diverse roles in CKD (Patel and Dressler, 2005). To elucidate the mechanisms underlying the reduction of viability and angiogenesis and the elevation of ROS production and apoptosis in iPSC-ECs treated with uremic toxin mixtures or uremic serum, we first determined whether uremic toxin mixtures induce activation of TGF- $\beta$ signaling in ECs. Phosphorylation levels of 8 TGF- $\beta$ pathway proteins were measured in iPSC-ECs after 


\section{Serum of normal controls and ESRD patients}

A

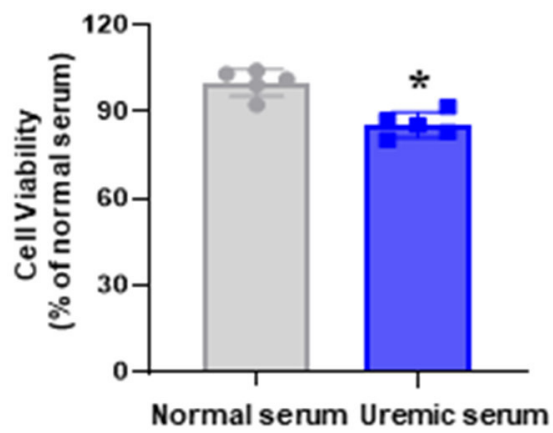

D

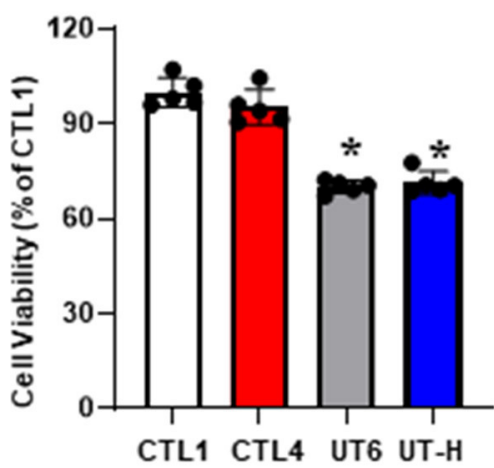

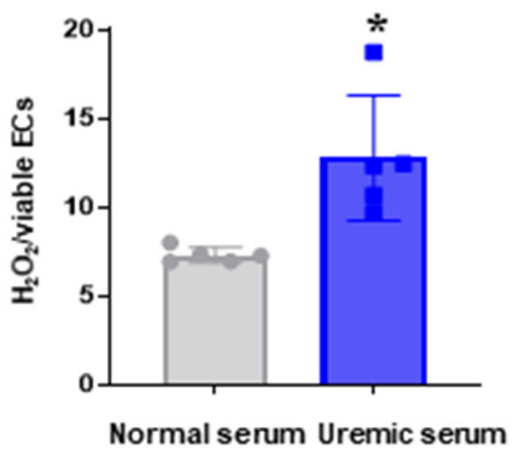

Normal serum Uremic serum

C

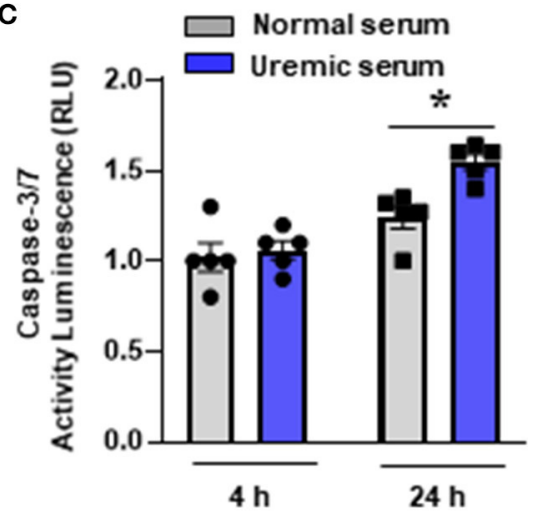

In vitro uremic toxins

E

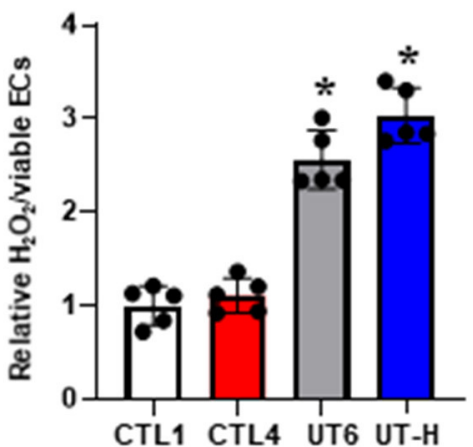

FIGURE 1 | The effects of uremic serum and in vitro uremic toxin mixtures on cell viability, ROS, and apoptosis of iPSC-ECs. (A) Uremic serum from ESRD patients significantly decreased the cell viability of IPSC-ECs. (B) ROS production increased in iPSC-ECs cultured with uremic serum. (C) Apoptosis of iPSC-ECs was enhanced by uremic serum at $24 \mathrm{~h}$. (D) UT6 and UT-H significantly decreased the cell viability of iPSC-ECs. (E) UT6 and UT-H significantly increased ROS production of iPSC-ECs. (F) UT6 and UT-H significantly increased apoptosis of iPSC-ECs. ${ }^{*} P<0.05$ compared to iPSC-ECs cultured with normal serum or CTL1. $n=5 /$ group. CTL, control; ESRD, end-stage renal disease; iPSC-ECs, induced pluripotent stem cells-derived endothelial cells; ROS, reactive oxygen species; UT6, uremic toxin mixture 6 (containing high concentrations of urea, creatinine, uric acid, indoxyl sulfate, and AGE); UT-H, uremic toxin mixture $\mathrm{H}$ (containing high concentrations of urea, creatinine, uric acid, and indoxyl sulfate).

treating cells with either CTL4 or UT-H (Figure 4A). We found that UT-H treatment significantly increased the phosphorylation of all 8 TGF- $\beta$ pathway proteins, ATF2, C-FOS, C-JUN, SMAD1, SMAD2, SMAD4, SMAD5, and TAK1, compared to CTL4 treatment (Figure 4B), suggesting that the inhibition of TGF- $\beta$ signaling may protect against uremic toxins.

In addition, to analyze the differences in humoral factors between normal serum and uremic serum, we measured the levels of 62 human inflammatory cytokines in serum from normal controls and ESRD patients (Supplementary Table 1). Of the 62 inflammatory cytokines measured, brain-derived neurotrophic factors (BDNF) and platelet-derived growth factor - BB (PDGF $\mathrm{BB})$ were significantly decreased in uremic serum compared with normal serum, whereas interleukin (IL)-12 p40, leptin, resistin, and vascular cell adhesion molecule 1 (VCAM1) were significantly increased in uremic serum (Figure 4C). In addition, most other cytokines including TGF- $\beta(P=0.057)$ demonstrated an increasing trend in uremic serum compared to normal serum (Supplementary Table 1). 
A

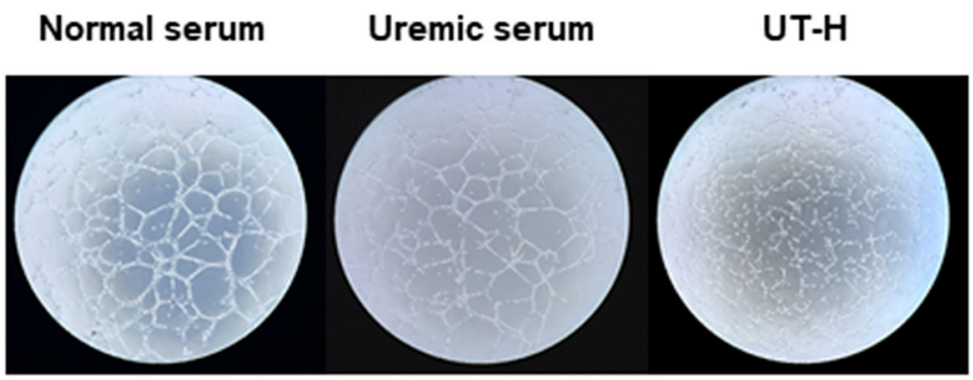

B
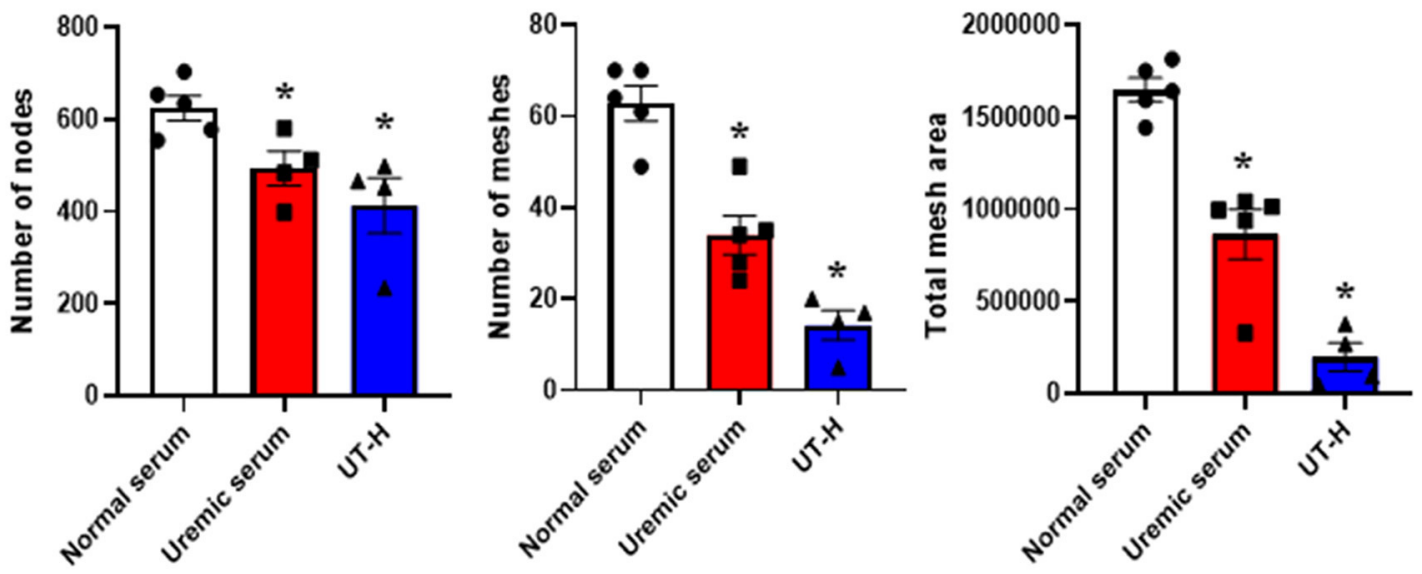

FIGURE 2 | The effects of uremic serum and in vitro uremic toxin mixture on vessel formation ability of iPSC-ECs. (A) Tube formation of iPSC-ECs was suppressed by uremic serum or UT-H. (B) Quantitative data from the tube formation assay. Uremic toxin mixtures exerted comparable effects with uremic serum on tube formation of iPSC-ECs. ${ }^{*} P<0.05$ compared to normal serum. $n=4-5 /$ group. UT-H, uremic toxin mixture $\mathrm{H}$ (containing high concentrations of urea, creatinine, uric acid, and indoxyl sulfate).

\section{Drug Screening Using a Simplified Uremic Vasculopathy Model With iPSC-ECs and Uremic Toxin Mixture}

We next sought to test whether the inhibition of TGF$\beta$ signaling reduces uremic toxin-induced impairment of endothelial function. iPSC-ECs were incubated with three drugs targeting the TGF- $\beta$ pathway, namely SD-208 (TGF- $\beta$ type 1 receptor inhibitor), losartan (TGF- $\beta$ signaling blocker), and LY2109761 (dual inhibitor of TGF- $\beta$ type 1 and 2 receptor inhibitor) before TGF- $\beta$ stimulation via treatment with uremic serum or UT-H. Treatment with SD-208 and LY2109761 significantly restored the formation of tubes in iPSC-ECs incubated with uremic serum and UT-H (Figure 5), which suggests that the inhibition of TGF- $\beta$ signaling is a protective mechanism against uremic vasculopathy.

To further validate our in vitro model as a drug screening model, we generated iPSCs from an ESRD patient and compared the newly generated ESRD patient-specific iPSC line against normal control iPSCs to determine whether patient-specific
iPSC-ECs can potentially recapitulate susceptibility against uremic vasculopathy. ESRD iPSCs exhibited typical expression of pluripotency markers (Supplementary Figure 1A). The differentiation efficiency and expression of endothelial markers of ESRD patient-specific iPSC-ECs were also comparable to those of normal control iPSC-ECs (Supplementary Figure 1B). The functional integrity of normal control and ESRD patient-specific iPSC-ECs was then compared by tube formation assay and migration assay. ESRD patient-specific iPSC-ECs showed impaired tube formation compared to normal control iPSC-ECs (Supplementary Figure 2). In the migration assay, the degree of wound healing was significantly lower in ESRD patient-specific iPSC-ECs (Figure 6A) compared to control iPSC-ECs. Interestingly, losartan and SD-208 significantly mitigated the impairment of wound healing in ESRD patient-specific iPSC-ECs (Figure 6B) demonstrating that patient-specific iPSC-ECs represent a model that may be used for personalized drug screening in the future. 
A

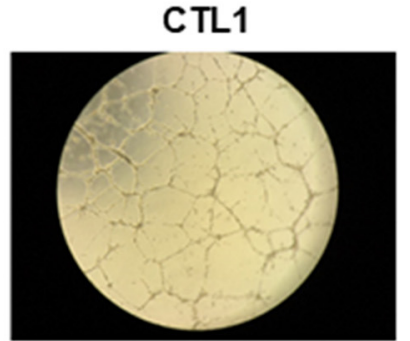

Creatinine

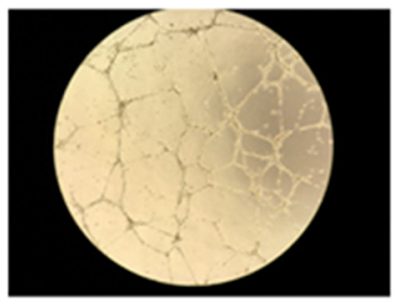

UT-H

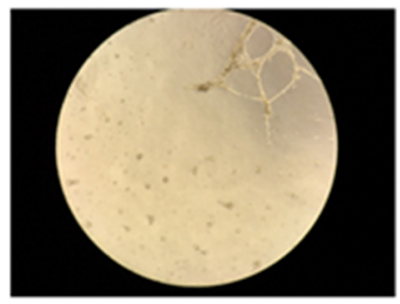

CTL4

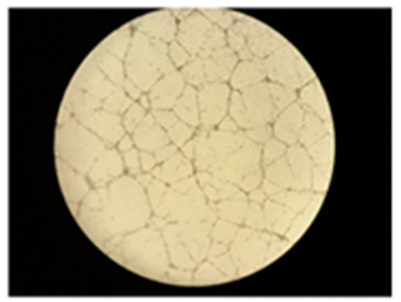

Uric acid
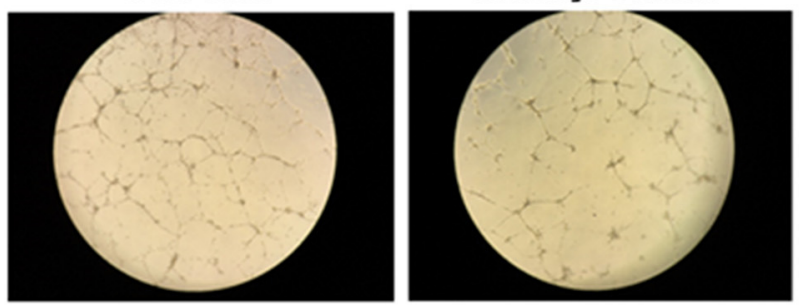

Urea

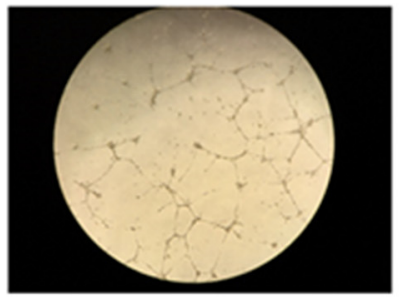

Indoxyl sulfate

B
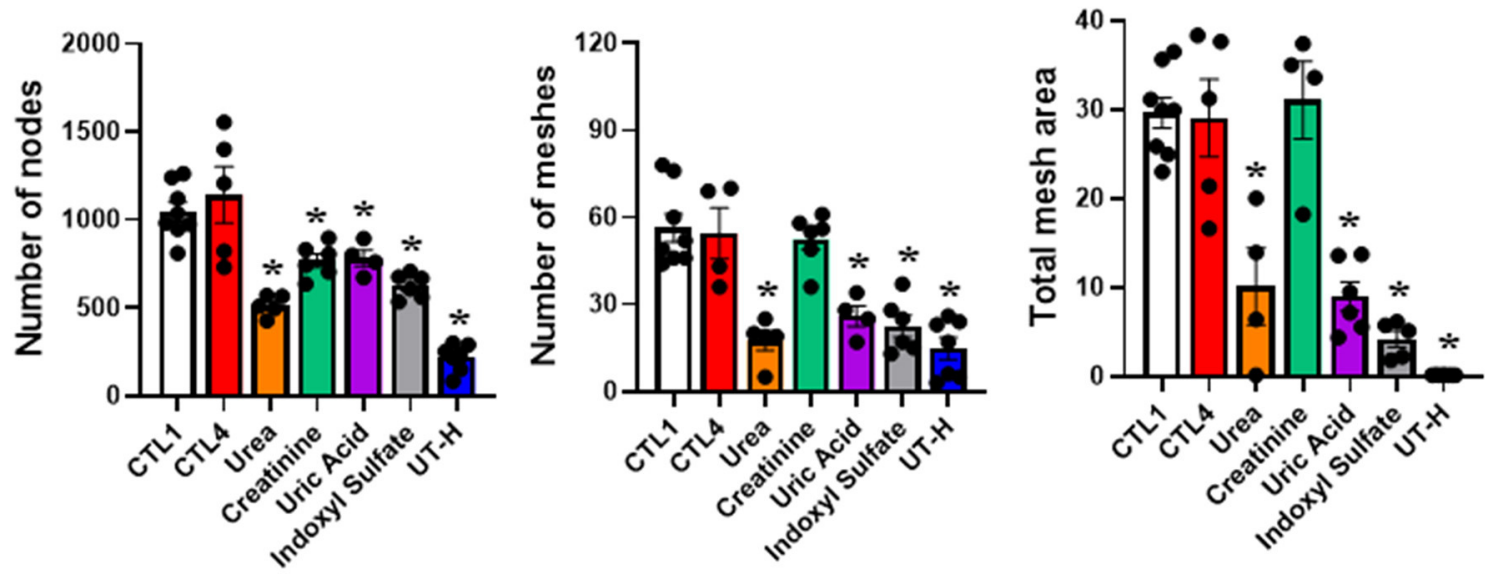

FIGURE 3 | The effects of each uremic toxin on vessel formation ability of iPSC-ECs. (A) Tube formation of iPSC-ECs was suppressed by each uremic toxin or UT-H. (B) Urea, uric acid, or indoxyl sulfate consistently suppressed tube formation of iPSC-ECs. ${ }^{\star} P<0.05$ compared to CTL1. $n=4-5 /$ group. CTL1, control 1; CTL4, control 4; UT-H, uremic toxin mixture $\mathrm{H}$ (containing high concentrations of urea, creatinine, uric acid, and indoxyl sulfate).

Gene and Functional Enrichment Analysis With the Addition of Uremic Toxin Mixture

Finally, to better understand the effects of UT-H on the transcriptome profiles of iPSC-ECs (GEO accession number: RNA-seq num GSE155969), RNA-sequencing was conducted.
Principal component analysis (PCA) was first conducted to examine the main source of variation in the data. PC1 reflected a closer correlation in iPSC-ECs from the same patient whereas PC3 identified distinct clusters corresponding to UT$\mathrm{H}$ treated control samples (Figure 7A). Hierarchical clustering 
A

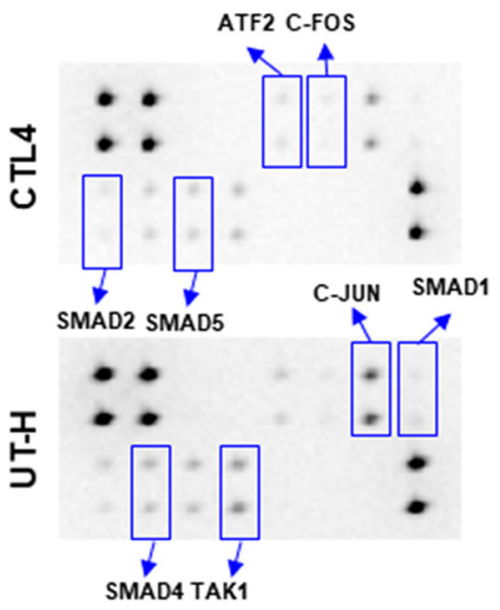

B

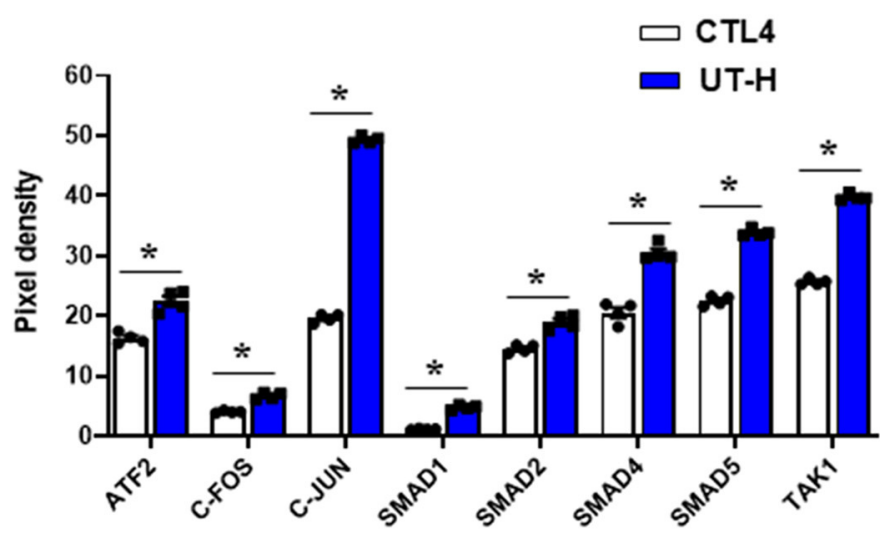

C

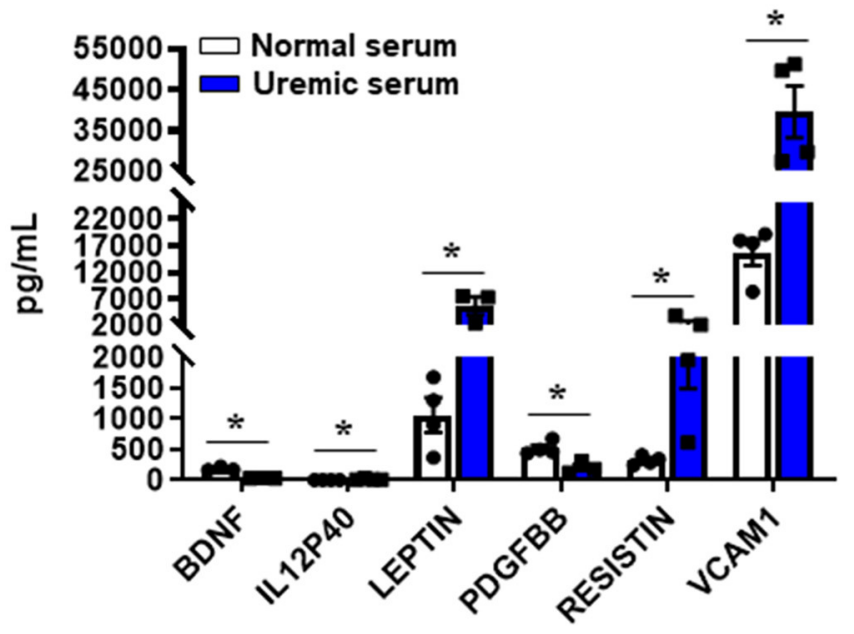

FIGURE 4 | The effects of uremic toxin mixtures on the intracellular TGF- $\beta$ pathway of iPSC-ECs and inflammatory cytokine levels in uremic serum. (A) Duplicated signals of 8 TGF- $\beta$ pathway phosphorylated proteins. (B) Quantitative and statistical analyses of each detected target. ${ }^{*} P<0.05$ compared to CTL4. $n=4 /$ group. CTL4, control 4 (media containing normal physiologic levels of urea, creatinine, and uric acid); UT-H, uremic toxin mixture $\mathrm{H}$ (containing high concentrations of urea, creatinine, uric acid, and indoxyl sulfate). (C) Luminex multiplex assay of normal and uremic serum. IL-12P40, leptin, resistin, and VCAM1 were higher in uremic serum than in normal serum. ${ }^{*} P<0.05$ compared to normal serum. $n=4$ /group. BDNF, Brain-derived neurotrophic factor; IL-12P40, interleukin-12 P40; PDGF BB, platelet-derived growth factor-BB; VCAM1, vascular cell adhesion molecule 1.

analysis (Figure 7B) of differentially expressed genes (DEGs) revealed different gene expression patterns after exposure to UT-H compared with control groups. In addition, a functional enrichment analysis was conducted to reveal the biological process and molecular function involved in a simplified in vitro uremic vasculopathy model. The most preserved expression profiles between control and UT-H treated samples were related to the amino acid transport across the plasma membrane, neutral amino acid transport, L-alpha-amino acid transmembrane transport, L-amino acid transport, as well as amino acid transmembrane transport (Figure 7C).

\section{DISCUSSION}

Complex and diverse systemic dysfunction involving nearly all major organs is common in ESRD patients, with cardiovascular complications being the leading cause of death (Tonelli et al., 
A

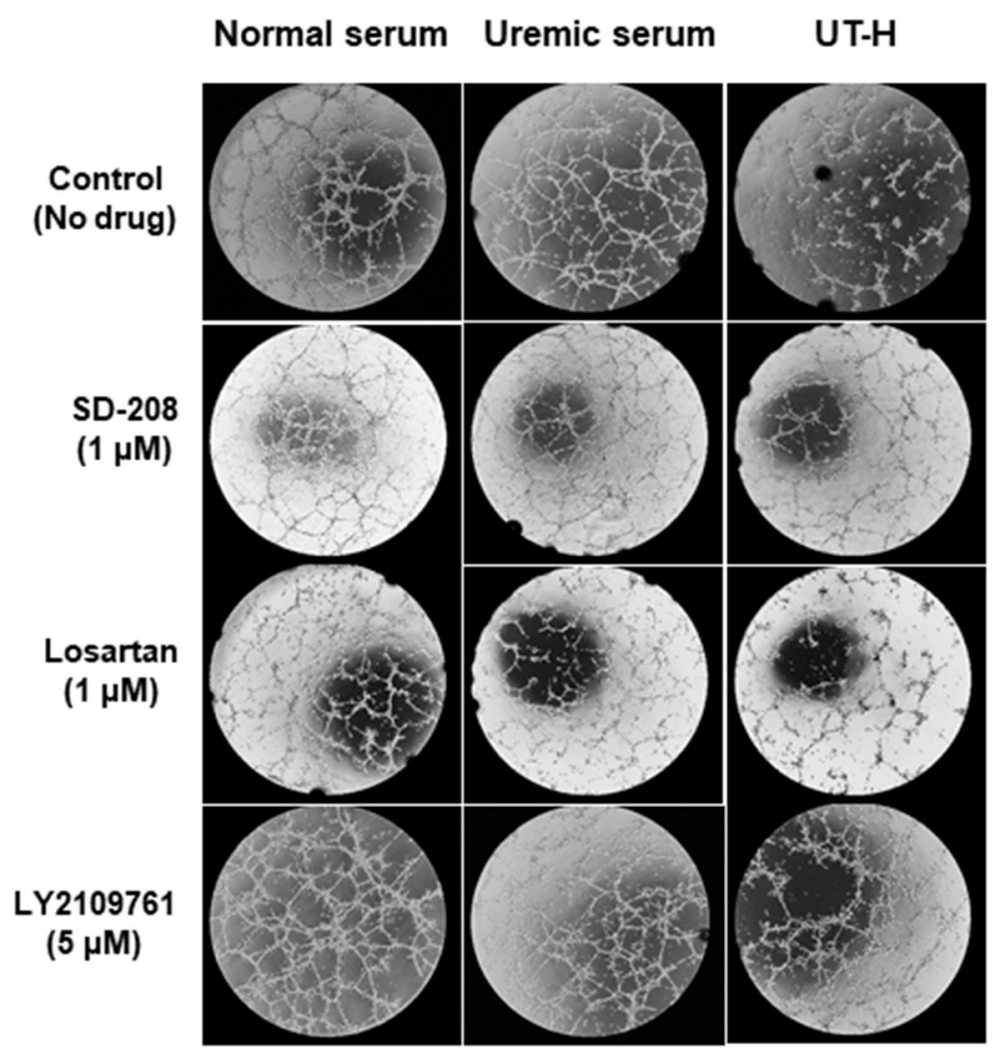

B

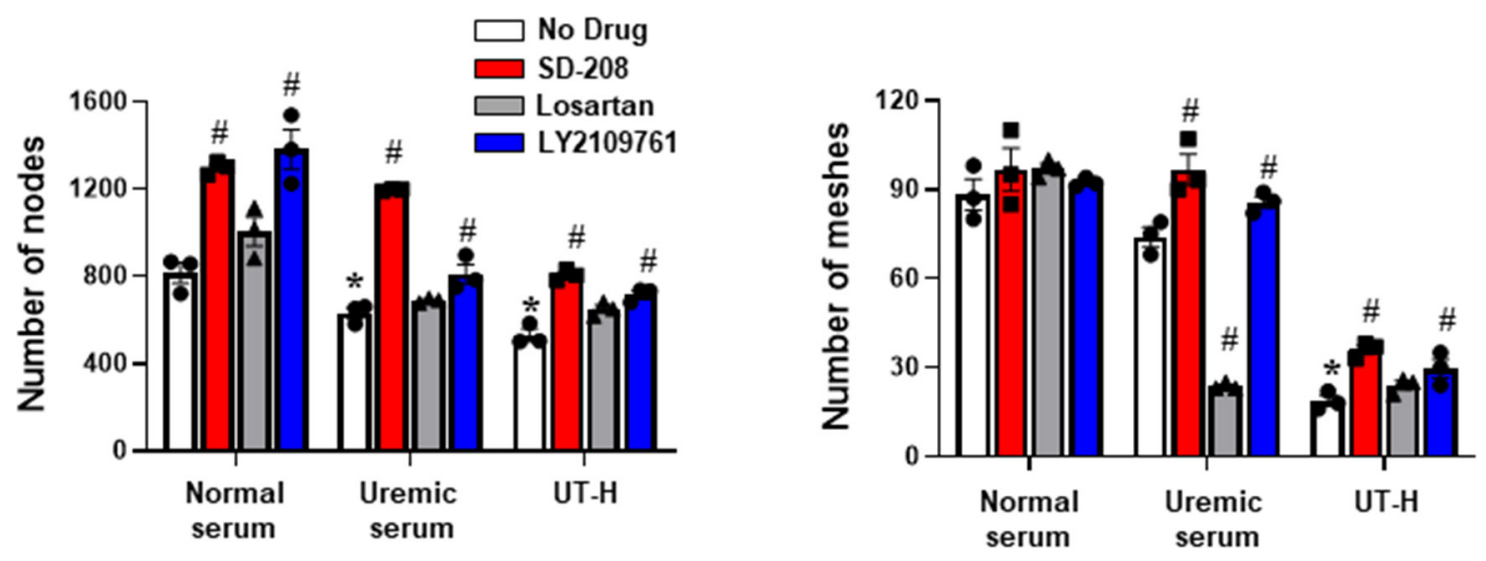

FIGURE 5 | Drug screening using a simplified uremic vasculopathy model. (A) The tube formation of iPSC-ECs was significantly suppressed in the uremic serum and UT-H groups. SD-208 and LY2109761 attenuated the adverse effects of uremic serum and UT-H on iPSC-ECs. The formation of capillary-like structures was imaged by phase-contrast microscopy (×20). (B) Quantitative data from the tube formation assay. Both SD-208 and LY2109761 showed favorable effects on the tube formation of iPSC-ECs treated with uremic serum or UT-H. Losartan also exerted favorable effects on iPSC-ECs treated with the UT-H but not significant. ${ }^{*} P<0.05$ compared to normal serum group with no drug. ${ }^{\#} P<0.05$ compared to no drug within the same group. $n=3 /$ group. UT-H, uremic toxin mixture $\mathrm{H}$ (containing high concentrations of urea, creatinine, uric acid, and indoxyl sulfate).

2006; Stenvinkel, 2010). Endothelial dysfunction is not only an initiating pathogenic factor but also the cornerstone in the progression of cardiovascular complications in CKD (AznarSalatti et al., 1995; Merino et al., 2008; Kramann et al., 2012).
Uremic vasculopathy is an accelerated atherosclerotic disease frequently accompanied by severe vascular calcification under the uremic micromilieu of CKD patients (Martin-Rodriguez et al., 2015; Guo et al., 2017). Although a few uremic toxins are 

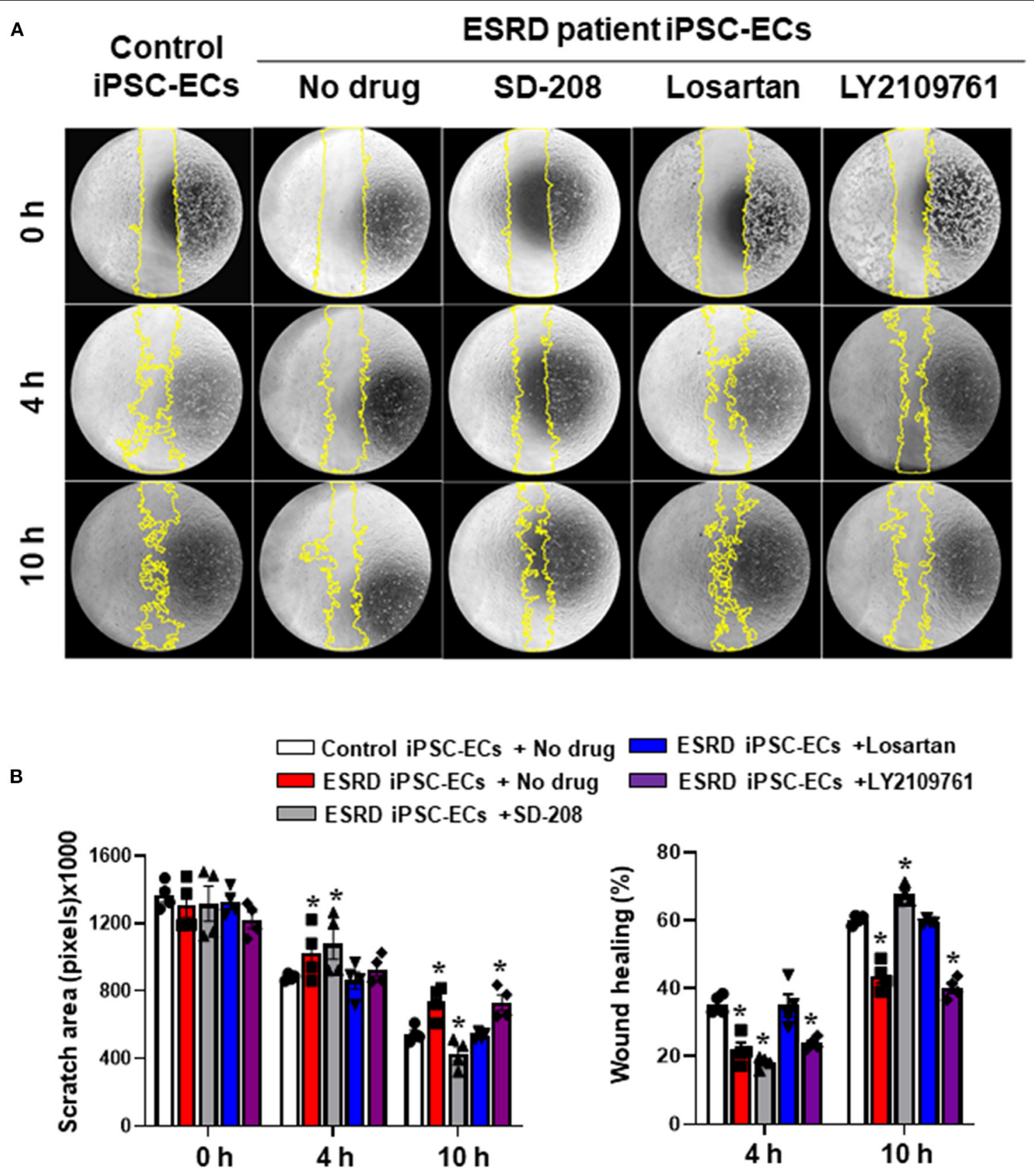

FIGURE 6 | Preliminary drug screening using ESRD patient-specific iPSC-ECs. (A) The degree of wound healing evaluated by a migration assay was significantly impaired in the ESRD patient-specific iPSC-ECs compared to the control iPSC-ECs. (B) Quantitative data from the migration assay. Losartan and SD-208 facilitated wound healing of the ESRD patient-specific iPSC-ECs. ${ }^{*} P<0.05$ compared to the control iPSC-ECs with no drug. Control iPSC-ECs, iPSC-ECs from a normal control; ESRD, end-stage renal disease; ESRD iPSC-ECs, iPSC-ECs from an ESRD patient receiving hemodialysis.

known to cause endothelial damage (Glorieux and Vanholder, 2011), the types or concentration of uremic toxins triggering uremic vasculopathy is not fully understood. In this study, we have demonstrated that an in vitro uremic milieu comprising of urea, creatinine, uric acid, and indoxyl sulfate leads to impairment of iPSC-EC functional integrity. Importantly, these effects were comparable to the effects of uremic serum collected from ESRD patients but are more reproducible and consistent.
We have also shown that this simplified uremic vasculopathy model is potentially amenable to drug screening by revealing that TGF- $\beta$ signaling inhibitors are capable of partially reversing the defects seen in iPSC-ECs exposed to uremic toxin mixtures. Lastly, we have shown that patient-specific iPSC-ECs derived from an ESRD patient exhibited a basal dysfunctional state compared to normal control iPSC-ECs, which can also be rescued by TGF- $\beta$ inhibitors. 
A
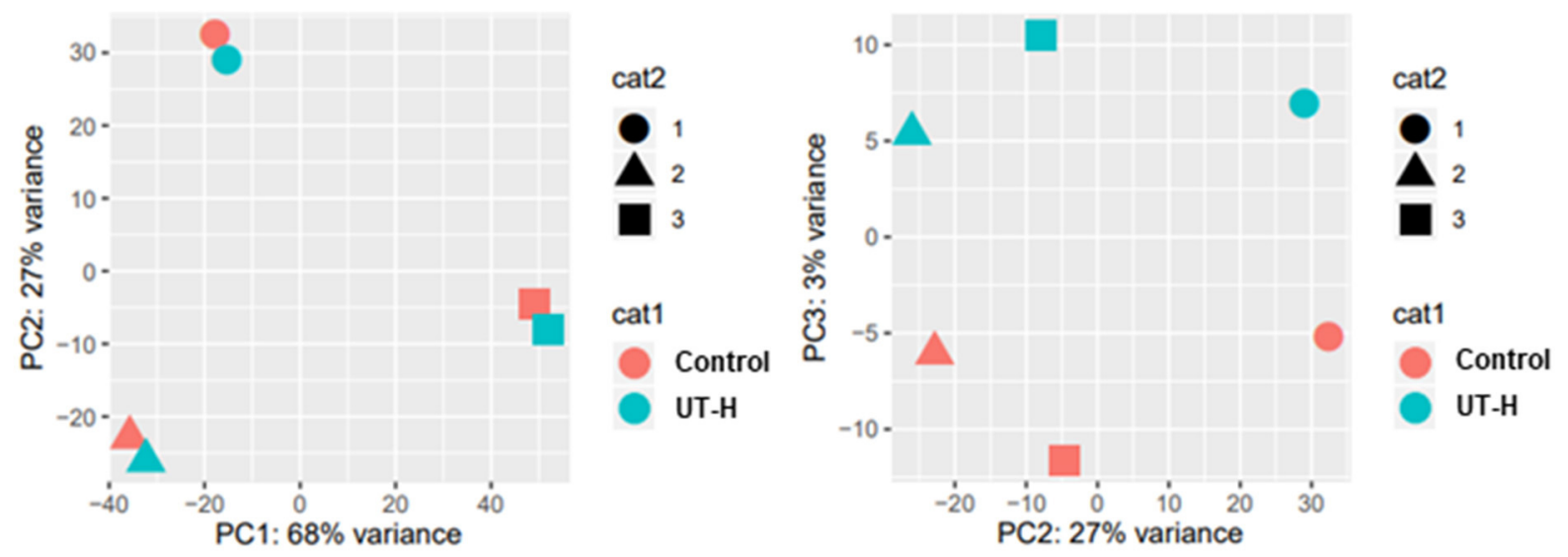

B

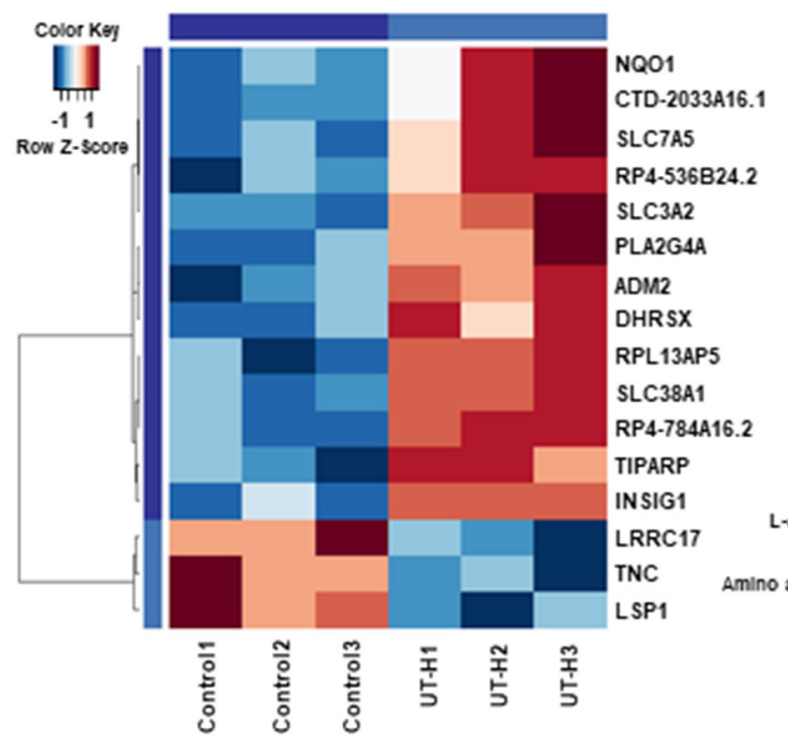

C

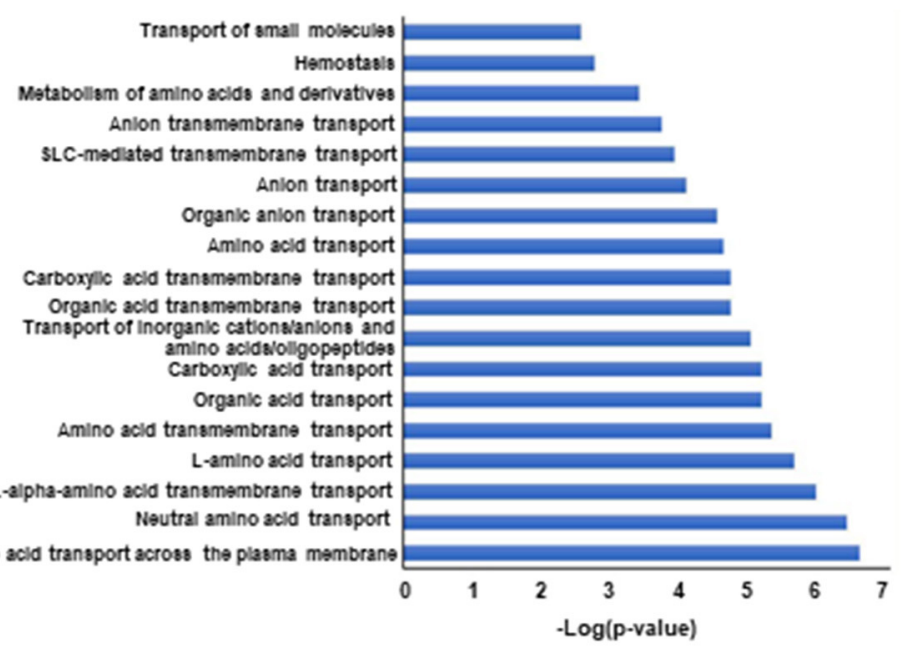

FIGURE 7 | Whole transcriptome analysis of treatment with uremic toxin mixture. (A) Principal component analysis (PCA) of gene expression profiles from indicated samples, shown as a scatter plot of PC1 vs. PC2 and PC2 vs. PC3. (B) Hierarchical clustering of differentially expressed genes (DEGs) between the UT-H treated group and control group. (C) Gene ontology (GO) pathway analysis of DEGs. UT-H, uremic toxin mixture (containing high concentrations of urea, creatinine, uric acid, and indoxyl sulfate)

Cell culture models are often used for simulating uremic vasculopathy in humans. For instance, human umbilical vein endothelial cells (HUVECs) are commonly exposed to $10-20 \%$ diluted serum of CKD patients (Chitalia et al., 2011; Lanza et al., 2015). HUVECs have been most frequently used in cell culture models of uremic vasculopathy. However, these cells often do not reflect disease susceptibility because of their unique characteristics mimicking stem cells such as hematopoietic support (Yamaguchi et al., 1998; Bal et al., 2012, 2013). iPSC-ECs in our study may be superior to HUVECs in reflecting genetic susceptibility to uremic vasculopathy. It remains a challenge to discern the effects of each uremic toxin per se on ECs using the serum of patients since serum usually contains a complex mixture of confounders such as cytokines and parathyroid hormones (Castillo-Rodríguez et al., 2017). In addition, differences in the concentration of each uremic toxin between individuals as well as temporal difference within the same patient also limit the usefulness of serum as a research tool. Moreover, there is also the ethical issue of acquiring large volumes of serum since most CKD patients are anemic. Based on these limitations, in this study, we investigated the effects of a simplified mixture of several well-known uremic toxins on iPSC-ECs, which were chosen by clinical criteria used to evaluate the degree of uremia as well as the pathogenic roles reported in previous studies. 
Urea and creatinine are typical uremic toxins, most commonly used to evaluate CKD patients in clinics (Lau and Vaziri, 2017). Urea substantially contribute to the progression atherosclerosis by inducing endothelial dysfunction, endothelial progenitor cells senescence, and apoptosis of vascular smooth muscle cells (Giardino et al., 2017). Uric acid is known as an important uremic toxin contributing to CKD progression (Gustafsson and Unwin, 2013; Tsai et al., 2017) and increasing risk of cardiovascular mortality in CKD patients (Luo et al., 2019). Urea, creatinine, and uric acid are widely used as essential parameters to evaluate CKD patients in nephrology clinics since these toxins are easily measurable under internationally standardized protocols. Indoxyl sulfate and AGE were reported to increase cardiovascular risk in CKD (Wang et al., 2005; Ito et al., 2013; Barisione et al., 2015). Indoxyl sulfate, the most representative gut-derived uremic toxin, shows endothelial toxicity and subsequently plays a substantial role in the progression of atherosclerosis by inhibiting endothelial proliferation and repair (Hung et al., 2017; Lano et al., 2020). Indoxyl sulfate also impaired the functional ability of human mesenchymal stem cells (Wang et al., 2016). Our data showed that a mixture of high concentrations of urea, creatinine, uric acid, and indoxyl sulfate can consistently mimic the detrimental effects of uremic serum on iPSC-ECs. Treatment with high concentrations of urea, uric acid, or indoxyl sulfate also significantly impaired tube formation of iPSC-ECs, supporting the vascular toxicity of these uremic toxins reported in previous studies. The physiologic concentrations of urea, creatinine, and uric acid on the other hand did not cause adverse effects on iPSC-ECs, supporting our hypothesis that a simplified mixture of highly concentrated urea, creatinine, uric acid, and indoxyl sulfate can exert adverse effects on iPSC-ECs mimicking uremic serum.

Using this in vitro uremic vasculopathy model, we successfully elucidated that impaired TGF- $\beta$ signaling is a potential pathogenic mechanism leading to impaired endothelial function in iPSC-ECs. This is in accordance with previous reports demonstrating that perturbed TGF- $\beta$ signaling induces endothelial-mesenchymal transition enhancing fibrosis and nonhereditary disorders such as atherosclerosis and cardiac fibrosis (Pardali et al., 2017; Goumans and Ten Dijke, 2018). Mutations in the TGF- $\beta$ superfamily have also been shown to disrupt endothelial patterning and induce arteriovenous malformations (Sugden and Siekmann, 2018). We also tested the feasibility of this simplified uremic vasculopathy model of iPSC-ECs for the screening of potential therapeutics. Losartan and TGF- $\beta$ inhibitors were chosen for drug screening based on the previous studies reporting cardiovascular protective effects of angiotensin receptor blockers in CKD (Kuriyama et al., 2006; Verbeke et al., 2014; Kim-Mitsuyama et al., 2018) and our data showing the activation of intracellular TGF- $\beta$ pathway in iPSC-ECs by in vitro uremic toxin mixtures. Both drugs attenuated the adverse effects of uremic toxins on normal control iPSC-ECs which is in line with a previous study reporting that the inhibition of TGF- $\beta$ signaling leads to the improved vascular network formation by iPSC-ECs (Kurokawa et al., 2017). As a further extension of our work, we also demonstrated that ESRD patient-specific iPSC-ECs revealed impaired wound healing ability compared to normal control iPSC-ECs demonstrating patient-specific iPSC-ECs recapitulate clinical susceptibility to a certain extent. Accordingly, the degree of wound healing was improved by losartan and SD-208 in ESRD iPSC-ECs. These results support the promising role of a simplified uremic vasculopathy model of iPSC-ECs as a novel drug screening tool reflecting disease susceptibility to uremic toxins.

Our study has a few limitations that should be addressed by future studies. First, more quantitative methodological approaches including functional genomics are required for improving the clinical usefulness of this model as a drug screening system. Although several quantitative or semiquantitative methods were used for analyzing phenotypic changes of iPSC-ECs in this study, more sensitive, as well as quantitative methods, are required for high throughput drug screening. Second, our study did not implement a standard method for evaluating the functional integrity of iPSC-ECs and there were some variations in evaluating the adverse effects of uremic toxins depending on the methods. A standardization process will be required for applying this approach in clinical practice. Third, we did not investigate aging effects on iPSC-ECs. Since the incidence of CKD and the risk of CKD progression to ESRD usually increase with age, further studies investigating differences in iPSC-ECs from young or old CKD patients would be required to improve clinical feasibility of this model.

\section{CONCLUSION}

Collectively, this study reports a novel platform of using iPSCECs to study uremic vasculopathy induced by a simplified uremic toxin mixture that can be applied as a potential drug screening system. Our data demonstrate that a simplified uremic toxin mixture can simulate the uremic micromilieu more reproducibly and consistently than uremic serum from ESRD patients. The deleterious effects of uremic toxins on iPSC-ECs were partly attenuated by an angiotensin-receptor blocker and TGF$\beta$ inhibitors supporting the potential usefulness of this model as a drug screening system. This new model of uremic vasculopathy represents a novel way to investigate the pathophysiology behind CKD and can be used to screen both conventional and new drugs.

\section{DATA AVAILABILITY STATEMENT}

The datasets generated for this study can be found in online repositories. The names of the repository/repositories and accession number(s) can be found at: NCBI GEO [accession: GSE155969].

\section{ETHICS STATEMENT}

The studies involving human participants were reviewed and approved by the Institutional Review Board of the Samsung Medical Center. The patients/participants provided their written informed consent to participate in this study. 


\section{AUTHOR CONTRIBUTIONS}

HRJ, HJC, WHL, and Y-GK wrote the first draft of the manuscript. HRJ, HJC, WH, S-GO, WHL, and Y-GK supervised the experiments. HRJ, YZ, N-YS, KL, JJ, JEL, and HL analyzed the data. All authors contributed to the article and approved the submitted version.

\section{FUNDING}

HRJ was supported by the Samsung Biomedical Research Institute (OTC 1180261), the National Research Foundation of the Republic of Korea grants funded by the Korean government (MSIT) (NRF-2016R1A2B4008235 \& 2019R1A2B5B01069346, NRF-2019R1A5A2027340), and the Korean Health Technology R\&D Project through the Korean Health Industry Development Institute (KHIDI), funded by the Ministry of Health \& Welfare, Republic of Korea (grant number: HI14C3484). KL was supported by a grant from the Korean Health Technology R\&D Project through the Korea Health Industry Development Institute (KHIDI), funded by the Ministry of Health \& Welfare, Republic of Korea (grant number: HI19C1337). S-GO was supported by the National Institutes of Health R00 HL130416 and R01 HL148756. WHL was supported by the American Heart Association Scientist Development Grant 16SDG27560003. N-YS was supported by the start-up research grant (SRG) from UM (SRG 2019-00177-FHS). WH was supported by the

\section{REFERENCES}

Aznar-Salatti, J., Escolar, G., Cases, A., Gómez-Ortiz, G., Anton, P., Castillo, R., et al. (1995). Uraemic medium causes endothelial cell dysfunction characterized by an alteration of the properties of its subendothelial matrix. Nephrol. Dial. Transplant. 10, 2199-2204. doi: 10.1093/ndt/10.12.2199

Bal, G., Kamhieh-Milz, J., Futschik, M., Haupl, T., Salama, A., and Moldenhauer, A. (2012). Transcriptional profiling of the hematopoietic support of interleukinstimulated Human Umbilical Vein Endothelial Cells (HUVECs). Cell Transplant. 21, 251-267. doi: 10.3727/096368911X580581

Bal, G., Kamhieh-Milz, J., Sterzer, V., Al-Samman, M., Debski, J., Klein, O., et al. (2013). Proteomic profiling of secreted proteins for the hematopoietic support of interleukin-stimulated human umbilical vein endothelial cells. Cell Transplant. 22, 1185-1199. doi: 10.3727/096368912X657288

Ballew, S. H., and Matsushita, K. (2018). Cardiovascular risk prediction in CKD. Semin. Nephrol. 38, 208-216. doi: 10.1016/j.semnephrol.2018.02.002

Baraka, A., and El Ghotny, S. (2012). Cardioprotective effect of renalase in 5/6 nephrectomized rats. J. Cardiovasc. Pharmacol. Ther. 17, 412-416. doi: $10.1177 / 1074248412446977$

Barisione, C., Ghigliotti, G., Canepa, M., Balbi, M., Brunelli, C., and Ameri, P. (2015). Indoxyl sulfate: a candidate target for the prevention and treatment of cardiovascular disease in chronic kidney disease. Curr. Drug Targets. 16, 366-372. doi: 10.2174/1389450116666141230114500

Bezenah, J. R., Kong, Y. P., and Putnam, A. J. (2018). Evaluating the potential of endothelial cells derived from human induced pluripotent stem cells to form microvascular networks in 3D cultures. Sci. Rep. 8:2671. doi: 10.1038/s41598-018-20966-1

Castillo-Rodriguez, E., Pizarro-Sanchez, S., Sanz, A. B., Ramos, A. M., SanchezNiño, M. D., Martin-Cleary, C., et al. (2017). Inflammatory cytokines as uremic toxins: “Ni Son Todos Los Que Estan, Ni Estan Todos Los Que Son”. Toxins 9:114. doi: 10.3390/toxins9040114
National Research Foundation of the Republic of Korea (NRF2017R1D1A1B04032172).

\section{ACKNOWLEDGMENTS}

We gratefully thank Kyoung Yi Choi, Jihyun Kang, Ji Woo Kim, and Woohyun Lee for technical assistance and Dr. Sekyung Oh and Dr. Jaecheol Lee for helpful advice.

\section{SUPPLEMENTARY MATERIAL}

The Supplementary Material for this article can be found online at: https://www.frontiersin.org/articles/10.3389/fcell.2020. 618796/full\#supplementary-material

Supplemental Figure 1 | iPSCs and iPSC-ECs of a normal control and an ESRD patient. (A) ESRD patient-specific iPSCs showed comparable expression of iPSC pluripotency markers, such as Nanog, COT4, TRA-1-60, and SSEA-4, compared to the normal control iPSCs. (B) ESRD patient-specific iPSC-ECs had a similar expression of endothelial markers including WWF, CD144 (VE-Cadherin), and CD31 compared to control iPSC-ECs. DAPI, 4',6-diamidino-2-phenylindole; ESRD, end-stage renal disease; OCT4, octamer-binding transcription factor 4; SSEA4, stage-specific embryonic antigen 4; TRA-1-60, podocalyxin; VE-Cadherin, vascular endothelial cadherin; WWF, von-Willebrand factor.

Supplementary Figure 2 | Tube formation of iPSC-ECs from a normal control and an ESRD patient. (A) Vessel structure formation ability of iPSC-ECs from a normal control and an ESRD patient was compared with tube formation. (B) Tube formation was significantly impaired in ESRD patient-specific iPSC-ECs compared to normal control iPSC-ECs. ${ }^{*} P<0.05$ compared to the control iPSC-ECs. ESRD, end-stage renal disease.

Chitalia, V. C., Murikipudi, S., Indolfi, L., Rabadi, L., Valdez, R., Franses, J. W., et al. (2011). Matrix-embedded endothelial cells are protected from the uremic milieu. Nephrol. Dial. Transplant. 26, 3858-3865. doi: 10.1093/ndt/gfr337

Churko, J. M., Burridge, P. W., and Wu, J. C. (2013). Generation of human iPSCs from human peripheral blood mononuclear cells using non-integrative Sendai virus in chemically defined conditions. Methods Mol. Biol. 1036, 81-88. doi: 10.1007/978-1-62703-511-8_7

Deppisch, R. M., Beck, W., Goehl, H., and Ritz, E. (2001). Complement components as uremic toxins and their potential role as mediators of microinflammation. Kidney Int. Suppl. 78, S271-S277. doi: 10.1046/j.1523-1755.2001.59780271.x

Giardino, I., D’Apolito, M., Brownlee, M., Maffione, A. B., Colia, A. L., Sacco, M., et al. (2017). Vascular toxicity of urea, a new "old player" in the pathogenesis of chronic renal failure induced cardiovascular diseases. Turk. Pediatri. Ars. 52, 187-193. doi: 10.5152/TurkPediatriArs.2017.6314

Glorieux, G., and Vanholder, R. (2011). New uremic toxins - which solutes should be removed? Contrib. Nephrol. 168, 117-128. doi: 10.1159/000321750

Go, A. S., Chertow, G. M., Fan, D., McCulloch, C. E., and Hsu, C. Y. (2004). Chronic kidney disease and the risks of death, cardiovascular events, and hospitalization. N. Engl. J. Med. 351, 1296-1305. doi: 10.1056/ NEJMoa041031

Goumans, M. J., and Ten Dijke, P. (2018). TGF- $\beta$ signaling in control of cardiovascular function. Cold Spring Harb. Perspect. Biol. 10:a022210. doi: $10.1101 /$ cshperspect.a022210

Guo, J., Lu, L., Hua, Y., Huang, K., Wang, I., Huang, L., et al. (2017). Vasculopathy in the setting of cardiorenal syndrome: roles of proteinbound uremic toxins. Am. J. Physiol. Heart Circ. Physiol. 313, H1-H13. doi: 10.1152/ajpheart.00787.2016

Gustafsson, D., and Unwin, R. (2013). The pathophysiology of hyperuricaemia and its possible relationship to cardiovascular disease, morbidity and mortality. BMC Nephrol. 14:164. doi: 10.1186/1471-2369-14-164 
Hung, S. C., Kuo, K. L., Wu, C. C., and Tarng, D. C. (2017). Indoxyl sulfate: a novel cardiovascular risk factor in chronic kidney disease. J. Am. Heart Assoc. 6:e005022. doi: 10.1161/JAHA.116.005022

Ito, S., Higuchi, Y., Yagi, Y., Nishijima, F., Yamato, H., Ishii, H., et al. (2013). Reduction of indoxyl sulfate by AST-120 attenuates monocyte inflammation related to chronic kidney disease. J. Leukoc. Biol. 93, 837-845. doi: $10.1189 /$ jlb.0112023

Jourde-Chiche, N., Dou, L., Cerini, C., Dignat-George, F., and Brunet, P. (2011). Vascular incompetence in dialysis patients-protein-bound uremic toxins and endothelial dysfunction. Semin. Dial. 24, 327-337. doi: 10.1111/j.1525-139X.2011.00925.x

Kim-Mitsuyama, S., Soejima, H., Yasuda, O., Node, K., Jinnouchi, H., Yamamoto, E., et al. (2018). Cardiovascular and renal protective role of angiotensin blockade in hypertension with advanced CKD: a subgroup analysis of ATTEMPT-CVD randomized trial. Sci. Rep. 8:3150. doi: 10.1038/s41598-018-20874-4

Kramann, R., Couson, S. K., Neuss, S., Floege, J., Knüchel, R., and Schneider, R. K. (2012). Uraemia disrupts the vascular niche in a 3D co-culture system of human mesenchymal stem cells and endothelial cells. Nephrol. Dial. Transplant. 27, 2693-2702. doi: 10.1093/ndt/gfr656

Kuriyama, S., Otsuka, Y., Uetake, D., Shirai, I., and Hosoya, T. (2006). Regression of cardiac hypertrophy using angiotensin II receptor blocker in patients with chronic kidney diseases. Nihon Jinzo Gakkai Shi. 48, 724-730.

Kurokawa, Y. K., Yin, R. T., Shang, M. R., Shirure, V. S., Moya, M. L., and George, S. C. (2017). Human induced pluripotent stem cell-derived endothelial cells for three-dimensional microphysiological systems. Tissue Eng. C Methods 23, 474-484. doi: 10.1089/ten.TEC.2017.0133

Lano, G., Burtey, S., and Sallée, M. (2020). Indoxyl sulfate, a uremic endotheliotoxin. Toxins 12:229. doi: 10.3390/toxins12040229

Lanza, D., Perna, A. F., Oliva, A., Vanholder, R., Pletinck, A., Guastafierro, S., et al. (2015). Impact of the uremic milieu on the osteogenic potential of mesenchymal stem cells. PLoS ONE 10:e116468. doi: 10.1371/journal.pone.0116468

Lau, W. L., and Vaziri, N. D. (2017). Urea, a true uremic toxin: the empire strikes back. Clin. Sci. 131, 3-12. doi: 10.1042/CS20160203

Lee, W. H., Ong, S. G., Zhou, Y., Tian, L., Bae, H. R., Baker, N., et al. (2019). Modeling cardiovascular risks of e-cigarettes with human-induced pluripotent stem cell-derived endothelial cells. J. Am. Coll. Cardiol. 73, 2722-2737. doi: 10.1016/j.jacc.2019.03.476

Levin, R. I., Kantoff, P. W., and Jaffe, E. A. (1990). Uremic levels of oxalic acid suppress replication and migration of human endothelial cells. Arteriosclerosis 10, 198-207.

Liao, Y., Smyth, G. K., and Shi, W. (2014). featureCounts: an efficient general purpose program for assigning sequence reads to genomic features. Bioinformatics 30, 923-930. doi: 10.1093/bioinformatics/btt656

Love, M. I., Huber, W., and Anders, S. (2014). Moderated estimation of fold change and dispersion for RNA-seq data with DESeq2. Genome Biol. 15:550. doi: 10.1186/s13059-014-0550-8

Luo, Q., Xia, X., Li, B., Lin, Z., Yu, X., and Huang, F. (2019). Serum uric acid and cardiovascular mortality in chronic kidney disease: a meta-analysis. BMC Nephrol. 20:18. doi: 10.1186/s12882-018-1143-7

Martin-Rodriguez, S., Caballo, C., Gutierrez, G., Vera, M., Cruzado, J. M., Cases, A., et al. (2015). TLR4 and NALP3 inflammasome in the development of endothelial dysfunction in uraemia. Eur. J. Clin. Invest. 45, 160-169. doi: 10.1111/eci.12392

Merino, A., Nogueras, S., Buendía, P., Ojeda, R., Carracedo, J., Ramirez-Chamond, R., et al. (2008). Microinflammation and endothelial damage in hemodialysis. Contrib. Nephrol. 161, 83-88. doi: 10.1159/000130412

Ong, S. B., Lee, W. H., Shao, N. Y., Ismail, N. I., Katwadi, K., Lim, M. M., et al. (2019). Calpain inhibition restores autophagy and prevents mitochondrial fragmentation in a human iPSC model of diabetic endotheliopathy. Stem Cell Rep. 12, 597-610. doi: 10.1016/j.stemcr.2019.01.017

Pardali, E., Sanchez-Duffhues, G., Gomez-Puerto, M. C., and Ten Dijke, P. (2017). TGF- $\beta$-induced endothelial-mesenchymal transition in fibrotic diseases. Int. J. Mol. Sci. 18:2157. doi: 10.3390/ijms18102157

Patel, S. R., and Dressler, G. R. (2005). BMP7 signaling in renal development and disease. Trends Mol. Med. 11, 512-518. doi: 10.1016/j.molmed.2005.09.007
Pertea, M., Kim, D., Pertea, G. M., Leek, J. T., and Salzberg, S. L. (2016). Transcriptlevel expression analysis of RNA-seq experiments with HISAT, stringTie and ballgown. Nat. Protoc. 11, 1650-1667. doi: 10.1038/nprot.2016.095

Sa, S., Gu, M., Chappell, J., Shao, N. Y., Ameen, M., Elliott, K. A., et al. (2017). Induced pluripotent stem cell model of pulmonary arterial hypertension reveals novel gene expression and patient specificity. Am. J. Respir. Crit. Care Med. 195, 930-941. doi: 10.1164/rccm.201606-12000C

Sallee, M., Dou, L., Cerini, C., Poitevin, S., Brunet, P., and Burtey, S. (2014). The aryl hydrocarbon receptor-activating effect of uremic toxins from tryptophan metabolism: a new concept to understand cardiovascular complications of chronic kidney disease. Toxins 6, 934-949. doi: 10.3390/ toxins6030934

Stenvinkel, P. (2010). Chronic kidney disease: a public health priority and harbinger of premature cardiovascular disease. J. Intern. Med. 268, 456-467. doi: 10.1111/j.1365-2796.2010.02269.x

Sugden, W. W., and Siekmann, A. F. (2018). Endothelial cell biology of Endoglin in hereditary hemorrhagic telangiectasia. Curr. Opin. Hematol. 25, 237-244. doi: 10.1097/MOH.0000000000000419

Takahashi, K., Tanabe, K., Ohnuki, M., Narita, M., Ichisaka, T., Tomoda, K., et al. (2007). Induction of pluripotent stem cells from adult human fibroblasts by defined factors. Cell 131, 861-872. doi: 10.1016/j.cell.2007.11.019

Tonelli, M., Wiebe, N., Culleton, B., House, A., Rabbat, C., Fok, M., et al. (2006). Chronic kidney disease and mortality risk: a systematic review. J. Am. Soc. Nephrol. 17, 2034-2047. doi: 10.1681/ASN.2005101085

Torres, P. A., and de Broe, M. (2012). Calcium-sensing receptor, calcimimetics, and cardiovascular calcifications in chronic kidney disease. Kidney Int. 82, 19-25. doi: 10.1038/ki.2012.69

Tsai, C. W., Lin, S. Y., Kuo, C. C., and Huang, C. C. (2017). Serum uric acid and progression of kidney disease: a longitudinal analysis and mini-review. PLoS ONE 12:e0170393. doi: 10.1371/journal.pone.0170393

Verbeke, F., Lindley, E., Van Bortel, L., Vanholder, R., London, G., Cochat, P., et al. (2014). A European Renal Best Practice (ERBP) position statement on the Kidney Disease: Improving Global Outcomes (KDIGO) clinical practice guideline for the management of blood pressure in non-dialysisdependent chronic kidney disease: an endorsement with some caveats for real-life application. Nephrol. Dial. Transplant. 29, 490-496. doi: 10.1093/ ndt/gft321

Wang, W., Liu, X., Wang, W., Li, J., Li, Y., Li, L., et al. (2016). The effects of indoxyl sulfate on human umbilical cord-derived mesenchymal stem cells in vitro. Cell. Physiol. Biochem. 38, 401-414. doi: 10.1159/000438639

Wang, Y., Marshall, S. M., Thompson, M. G., and Hoenich, N. A. (2005) Cardiovascular risk in patients with end-stage renal disease: a potential role for advanced glycation end products. Contrib. Nephrol. 149, 168-174. doi: 10.1159/000085483

Xie, J., Yoon, J., An, S. W., Kuro-o, M., and Huang, C. L. (2015). Soluble Klotho protects against uremic cardiomyopathy independently of fibroblast growth factor 23 and phosphate. J. Am. Soc. Nephrol. 26, 1150-1160. doi: 10.1681/ASN.2014040325

Yamaguchi, H., Ishii, E., Tashiro, K., and Miyazaki, S. (1998). Role of umbilical vein endothelial cells in hematopoiesis. Leuk. Lymphoma. 31, 61-69. doi: 10.3109/10428199809057585

Zhou, Y., Zhou, B., Pache, L., Chang, M., Khodabakhshi, A. H., Tanaseichuk, O., et al. (2019). Metascape provides a biologist-oriented resource for the analysis of systems-level datasets. Nat. Commun. 10:1523. doi: 10.1038/s41467-019-09234-6

Conflict of Interest: The authors declare that the research was conducted in the absence of any commercial or financial relationships that could be construed as a potential conflict of interest.

Copyright (C) 2021 Jang, Cho, Zhou, Shao, Lee, Le, Jeon, Lee, Huh, Ong, Lee and Kim. This is an open-access article distributed under the terms of the Creative Commons Attribution License (CC BY). The use, distribution or reproduction in other forums is permitted, provided the original author(s) and the copyright owner(s) are credited and that the original publication in this journal is cited, in accordance with accepted academic practice. No use, distribution or reproduction is permitted which does not comply with these terms. 